\title{
Enfermedades transmitidas por Alimentos (Etas); Una Alerta para el Consumidor
}

Sergio Fernández

sfernandez16-0510@unag.edu.hn Facultad de Ciencias Tecnológicas, Universidad Nacional de Agricultura, Catacamas, Olancho, Honduras

Jhunior Marcía

jmarcia@unag.edu.hn

Facultad de Ciencias Tecnológicas, Universidad Nacional de Agricultura, Catacamas, Olancho,

Honduras

Jessy Bu

jbu19a0433@unag.edu.hn

Facultad de Ciencias Tecnológicas, Universidad Nacional de Agricultura, Catacamas, Olancho,

Honduras

Yanina Baca

yanina.baca@unah.edu.hn

Facultad de Ciencias Químicas y Farmacia, Universidad Nacional Autónoma de Honduras,

Tegucigalpa, Honduras

Vilma Chavez

vpchavez@unah.hn

Facultad de Ciencias Químicas y Farmacia, Universidad Nacional Autónoma de Honduras,

Tegucigalpa, Honduras

Hector Montoya

hector.montoya@unah.hn

Ingris Varela

vareingris87@gmail.com

Facultad de Ciencias Tecnológicas, Universidad Nacional de Agricultura, Catacamas, Olancho,

Honduras

Jenny Ruiz

cardona15jenny@gmail.com

Facultad de Ingeniería Agroindustrial, Universidad Nacional Autónoma de Honduras, Juticalpa,

Honduras

Suany Lagos

lagosuany98@gmail.com

Facultad de Ingeniería Agroindustrial, Universidad Nacional Autónoma de Honduras, Juticalpa, Honduras

Franklin Ore

franklin.ore@unh.edu.pe

Facultad de Ciencias Agrarias, Universidad Nacional de Huancavelica, Perú 


\section{RESUMEN}

Las Enfermedades Transmitidas por los Alimentos (ETAs) son provocadas por la ingestión de alimentos contaminados por microorganismos o sustancias químicas, y representan una importante carga de mortalidad y morbilidad dentro de los sistemas de Salud Pública de las naciones, impactando igualmente en el comercio internacional. Se clasifican en infecciones alimentarias e intoxicaciones alimentarias, y sus agentes causales pueden ser de tipo químicos, físicos o biológicos. En el mundo 1 de cada 10 personas se enferman por ingerir alimentos contaminados, causando un total de 420 mil muertes anuales, de las que 125 mil representan a niños. Más del 70\% de los casos de ETA's se originan debido a una manipulación inadecuada, por ende, se recomienda utilizar las Buenas Prácticas Agrícolas y Buenas Practicas de Manufactura como medidas de prevención.

Palabras claves: infecciones, intoxicaciones alimentarias, alimentos contaminados, enfermedades transmitidas por alimentos. 


\title{
Foodborne diseases (etas); a consumer alert
}

\begin{abstract}
Foodborne Diseases are caused by the ingestion of food contaminated by microorganisms or chemical substances, and represent a significant burden of mortality and morbidity within the Public Health systems of nations, also impacting international trade. They are classified as food infections and food poisoning, and their causative agents can be chemical, physical or biological. In the world, 1 in 10 people get sick from eating contaminated food, causing a total of 420 thousand deaths annually, of which 125 thousand represent children. More than 70\% of the cases of Foodborne Diseases originate due to improper handling, therefore, it is recommended to use Good Agricultural and Good Manufacturing Practices as prevention measures.
\end{abstract}

Keywords: infections, food poisoning, contaminated food, foodborne illness.

Artículo recibido: 27 marzo 2021

Aceptado para publicación: 30 abril 2021

Correspondencia: sfernandez16-0510@unag.edu.hn

Conflictos de Interés: Ninguna que declarar 


\section{INTRODUCCION}

Se dice que ocurre contaminación alimentaria cuando existe la presencia de cualquier materia anormal en el alimento que conlleve a la pérdida de su calidad, ya sea para el consumo humano o animal (Barreto et al., 2010). Se han descrito más de 250 tipos de ETAs debido a la naturaleza amplia y heterogenia de los contaminantes, los cuales pueden ser químicos; físicos y biológicos (WHO, 2002; Rosas, 2007). Dentro de estos últimos se encuentra una amplísima gama de bacterias, en general responsables del mayor número de ETAs en los países en vías de desarrollo (Rosas, 2007).

Según cifras que presenta la Organización Mundial de la Salud (OMS, 2015) cada año hasta 600 millones de personas de todo el mundo, o casi 1 de cada 10, enferman tras consumir alimentos contaminados, de las cuales 420,000 mueren, incluidos 125,000 niños menores de 5 años. En Honduras cada persona sufre de gastroenteritis causadas por ETAs cerca de 3,5 veces por año (FAO, 2009), lo cual es 17 veces mayor, si se compara con países como los Estados Unidos que presenta menos de 0,2 casos por individuo por año (Mead et al., 1999).

La finalidad de este artículo es presentar una revisión literaria sobre las enfermedades transmitidas por los alimentos a partir de fuentes prestigiosas e información propia, que reúna información acerca de los aspectos generales como su definición, causas y tipos de ETAs, impacto global y nacional, alimentos de mayor riesgo y prevención de las mismas.

\section{Enfermedades transmitidas por los alimentos (ETAs)}

Las ETAs se producen por la ingestión de alimentos y/o bebidas contaminados con microorganismos patógenos que afectan la salud del consumidor en forma individual o colectiva (Gonzales \& Rojas, 2005) y son consideradas como una importante carga de enfermedad en el mundo (Olea et al., 2012). Estas enfermedades se caracterizan por una variedad de síntomas gastrointestinales, como náuseas, vómito, diarrea, dolor abdominal y fiebre; en algunos casos se pueden presentar complicaciones severas, como sepsis, meningitis, abortos, síndrome de Reiter, síndrome de Guillan Barré o la muerte (Linscott, 2011; Steniner, 2013; INS, 2018).

La Organización Mundial de la Salud (OMS) señala que en los países menos desarrollados, las ETAs son la principal causa de enfermedad y muerte, asociadas a una carga socioeconómica significativa (OMS, 2015; Hall et al., 2006), mientras que Roberts \& Sockett (1994), mencionan que en los países desarrollados, las ETAs son responsables de altos niveles de pérdida de productividad, costos asociados al uso de los servicios de salud y a la implementación y monitoreo de políticas de inocuidad de los alimentos. 
Aproximadamente $70 \%$ de las diarreas se originan por la ingestión de alimentos contaminados con microorganismos o toxinas (Zúñiga \& Caro, 2017). Son alrededor de 250 agentes causantes de ETAs, entre los que se incluyen bacterias, virus, hongos, parásitos, priones, toxinas y metales (Jones et al., 2008; Afzal et al., 2011; Linscott, 2011; Nyenje \& Ndip, 2013). Los cambios en los hábitos alimentarios de la sociedad, como el consumo de alimentos envasados, comidas fuera del hogar, expendio de comidas preparadas y comidas rápidas, son factores que contribuyeron al incremento de las ETAs (Olea et al., 2012; Gonzales \& Rojas, 2005).

\section{Causas de las ETAs}

La contaminación con agentes patógenos y su posterior multiplicación incontrolada, representa las causas más frecuentes de las enfermedades de transmisión alimentaria (Vásquez, 2003). En la mayoría de los casos son consecuencia de un tratamiento incorrecto de los alimentos durante su obtención, transformación, almacenamiento o preparación (Domínguez \& Oliver, 2007). De acuerdo a la Organización Panamericana de la Salud (OPS, 2019), los contaminantes de los alimentos pueden ser químicos (compuestos inorgánicos tóxicos, antimicrobianos, promotores del crecimiento, aditivos alimentarios tóxicos, lubricantes y tintas, toxinas naturales, desinfectantes, metales pesados, pesticidas y sustancias empleadas en agricultura que no pueden eliminarse con un lavado, o se han sometido al mismo de forma insuficiente), físicos (fragmentos de vidrio, metal, madera, u otros que puedan ocasionar daño al consumidor) o biológicos (bacterias, parásitos, e incluso abióticos como virus y priones).

La mayoría de las ETAs tienen una etiología viral, sin embargo, el mayor número de hospitalizaciones y defunciones son provocadas por una amplísima gama de agentes bacterianos responsables de las denominadas toxiinfecciones alimentarias (TIA) (Teplitski et al., 2009; Sedrés et al., 2009; Barreto et al., 2009, 2010; Rodríguez et al., 2009, 2011a). Las TIA se manifiestan poco después (horas o días) de haber consumido alimentos o bebidas no aptas a ese fin por estar contaminados con microorganismos o sus toxinas (Rodríguez et al., 2015).

\section{Tipos de ETAs}

Las ETAs pueden ser de dos tipos: Infecciones Alimentarias e Intoxicaciones Alimentarias (de la Hoz, 2016). Una infección alimentaria ocurre cuando se ingieren alimentos que contienen microorganismos patógenos vivos (García, 2020). En general, son determinadas por la invasión, multiplicación y alteraciones de los tejidos del huésped producidas por gérmenes 
transportados por los alimentos. Las infecciones alimentarias presentan dos variantes: $a$ ) infecciones invasivas: se caracterizan por la colonización de tejidos y órganos provocadas por el agente causal. Este grupo comprende virus, protozoos parásitos y bacterias como Salmonella, Aeromonas, Campylobacter, Shigella, Vibrio parahaemolyticus, Yersinia y Escherichia coli enteroinvasivas (EIEC) (Riverón, 1999; Schmidt et al., 2003; Barreto, 2007). b) Toxiinfecciones: ocasionadas por bacterias no invasivas, pero capaces de colonizar y multiplicarse en el tracto intestinal del hospedero, donde excretan sus toxinas, tal es el caso de: Vibrio cholerae, Bacillus cereus (cepas productoras de enterotoxinas), Clostridium botulinum, Clostridium perfringens y las variantes enteropatógenas de E. coli productoras de enterotoxinas, verotoxinas, o ambas (Schmidt et al., 2003; Barreto, 2007), las cuales necesitan de ciertos umbrales para general la toxiinfección (Tabla 1).

Por otra parte, las intoxicaciones alimentarias son producidas por la ingestión de toxinas formadas en tejidos de plantas o animales, de productos metabólicos excretados por microorganismos en los alimentos, o por sustancias químicas que se incorporan a ellos de modo accidental, incidental o intencional en cualquier momento desde su producción hasta su consumo (Pinillos et al., 2003). Las intoxicaciones, en general, se manifiestan más rápidamente, que las infecciones alimentarias. En las infecciones alimentarias el agente se potencia numéricamente en el consumidor, mientras que en las intoxicaciones alimentarias prevalece en los alimentos (Rodríguez et al., 2015).

Tabla 1. Dosis umbrales para generar toxiinfección

\begin{tabular}{|c|c|}
\hline Microorganismos & Dosis umbral (Bacterias/G) \\
\hline Salmonelas & $10^{5}-10^{6}$ \\
\hline Shigelas & $10^{3}-10^{4}$ \\
\hline Vibrio parahemolítico & $10^{5}-10^{6}$ \\
\hline Clostridium perfringens & $10^{6}$ \\
\hline Bacillus cereus & $10^{8}-10^{6}$ \\
\hline Estafilococos & $10^{6}-10^{9}$ \\
\hline
\end{tabular}

Fuente: Rosas (2007). 


\section{Impacto global y nacional de las ETAs}

Según la Organización Mundial de la Salud la incidencia anual de diarreas es de 1500 millones de casos, y 3 millones de niños menores de cinco años mueren anualmente (Olea $e t$ al., 2012). La presencia de contaminación alimenticia ya sea mediante intoxicaciones o infecciones bacterianas, parasitarias o una combinación de las mismas (infecto-intoxicación) son frecuentes y afectan principalmente a grupos sociales de bajos recursos, como ya se mencionó, quienes por razones económicas, la mayoría de las veces tienen acceso a alimentos de bajo costo, cuya calidad e inocuidad en muchos casos es por lo menos dudosa (Zuñiga \& Caro, 2017).

La carga mundial muestra que casi 1 de cada 10 personas enferman cada año al ingerir alimentos contaminados y 420,000 mueren como consecuencia de estas enfermedades (OMS, 2015). Sobre la base de la mortalidad infantil y de adultos, 14 países tienen diferentes cargas de eta, con la mayor proporción en las subregiones de África, seguidas de las subregiones en el sudeste de Asia y la subregión del Mediterráneo Oriental (Havelaar et al., 2015; Gutiérrez, 2009).

De acuerdo a la FAO (2009), el promedio anual de infecciones bacterianas en Honduras corresponde a cerca del 99 por ciento de los casos informados, los virus son cerca del uno por ciento y los casos por agentes químicos, incluyendo toxinas no bacterianas, no alcanzan al 0,1 por ciento (Tabla 2). En un estudio específico, de los casos de etiología conocida se informó de 92 por ciento por agentes bacterianos, cinco por ciento por agentes virales, dos por ciento por agentes químicos y menos del uno por ciento por parásitos (Bean et al., 1990). Las diferencias con otros países podrían deberse en parte a una identificación más eficaz en los países desarrollados y a una mayor tasa de pacientes que en Honduras, por razones socioeconómicas, no acuden al médico.

El hondureño medio sufre de gastroenteritis causadas por enfermedades transmitidas por alimentos cerca de 3,5 veces por año. Si se compara con el promedio estimado en 1999 en Estados Unidos de América de menos de 0,2 casos por individuo por año (Mead et al., 1999), es considerablemente alto. A pesar de los esfuerzos del gobierno, la empresa privada y las ONG para mejorar la inocuidad de los alimentos en el marco de la globalización y la salud pública, evidentemente es necesario mejorar el sistema actual. 
Tabla 2. Estimación de la incidencia anual de enfermedades transmitidas por alimentos en Honduras (Tomado de FAO, 2009)

\begin{tabular}{|c|c|c|c|c|c|}
\hline Agente causal & $\underset{1}{\text { Informado }}$ & $\begin{array}{c}\% \text { por } \\
\text { alimentos }^{2}\end{array}$ & $\begin{array}{c}\text { Factor de } \\
\text { extrapolación }\end{array}$ & Estimación & $\%$ \\
\hline Bacterianas & $256,915.9$ & 80 & 4,36 & $4,425,584$ & 21,16 \\
\hline $\begin{array}{c}\text { Fiebre tifoidea y } \\
\text { paratifoidea }\end{array}$ & 85,5 & 95 & 38,59 & 234,878 & 1,12 \\
\hline $\begin{array}{l}\text { Otras infecciones } \\
(\text { Salmonella sp.) }\end{array}$ & 7,7 & 20 & 38,59 & 187,397 & 0,90 \\
\hline Shigelosis & 4,3 & 70 & 20,44 & 104,526 & $\mathbf{0 , 5 0}$ \\
\hline $\begin{array}{c}\text { Otras infecciones } \\
\text { intestinales bacterianas }\end{array}$ & 300,5 & 2 & 4,36 & $3,873,233$ & 18,52 \\
\hline $\begin{array}{c}\text { Carbunco (Bacillus } \\
\text { anthracis) }\end{array}$ & 1,2 & 99 & 4,36 & 3,292 & 0,02 \\
\hline Listeriosis & 8,1 & & & 22,258 & 0,11 \\
\hline $\begin{array}{c}\text { Diarrea gastroenteritis } \\
\text { de presunto } \\
\text { origen infeccioso }\end{array}$ & 256508,6 & & & & \\
\hline Parasitarias & 115,0 & & 260 & $2,668,024$ & 12,75 \\
\hline Amebiasis & 76,3 & 10 & 200,000 & 1,984 & 0,01 \\
\hline $\begin{array}{l}\text { Otras enfermedades } \\
\text { intestinales } \\
\text { (protozoarios) } \\
\end{array}$ & 26,3 & 50 & 24000 & 2630000 & 12,57 \\
\hline Toxoplasmosis & 3 & 50 & 4,36 & 36000 & 0,17 \\
\hline $\begin{array}{c}\text { Otras infecciones } \\
\text { debidas a } \\
\text { trematodos }\end{array}$ & 2,4 & 90 & 4,36 & 9 & $<0,01$ \\
\hline Cisticercosis & 7 & 100 & & 30 & $<0,01$ \\
\hline Virus & 2548,0 & & & $13,833,653$ & 66,10 \\
\hline $\begin{array}{l}\text { Infecciones intestinales } \\
\text { (virus y otros) }\end{array}$ & 62,2 & 40 & 661,000 & $13,445,680$ & 66,10 \\
\hline Hepatitis aguda tipo A & $2,485.8$ & 5 & 3 & 373 & $<0,01$ \\
\hline Toxinas & 39,8 & & & 808 & $<0,01$ \\
\hline $\begin{array}{l}\text { Otras intoxicaciones } \\
\text { alimentarias } \\
\text { bacterianas }\end{array}$ & 30,3 & 100 & 20,34 & 616 & $<0,01$ \\
\hline $\begin{array}{l}\text { Sustancias nocivas } \\
\text { ingeridas como } \\
\text { alimento }\end{array}$ & 9,5 & 100 & 20,16 & 192 & $<0,01$ \\
\hline Total & 259618,7 & & & 20928069 & 100,0 \\
\hline
\end{tabular}

${ }^{1}$ Promedio de los informes de seguimiento pasivo de la Secretaría de Salud, años 1998, 1999, 2001-2006 y el IHSS, años 1991-1995, 1997-2003.

${ }^{2}$ Porcentaje estimado en que el vehículo del agente causal es un alimento, basado en estudios previos (Más-Coma, 1999; Mead et al., 1999; Schneider et al., 2004).

\section{Alimentos de mayor riesgo}

Las características intrínsecas de los alimentos son determinantes en la contaminación y proliferación de las bacterias, lo cual genera una diversidad de alimentos asociados a las ETAs (Rodríguez et al., 2015). El instituto de Tecnología Alimentaria (IFT, 2004) menciona 
que la carne, el pescado, los huevos, la leche y sus derivados (Tabla 3), son considerados de alto riesgo por su alto contenido proteico. Mientras que Rosas (2007), asocia la actividad del agua de los alimentos como un factor determinante para el crecimiento de los microorganismos, y cualquier tratamiento que la reduzca contribuye a la preservación de estos productos.

Por otro lado, los alimentos que requieren considerable manipulación para su elaboración, y luego se guardan a elevada temperatura, como ocurre en los países tropicales, son los candidatos por excelencia en las intoxicaciones, de ahí la alta frecuencia de $S$. aureus en los productos de repostería, lo cual no excluye su presencia en otros como cárnicos, ensaladas y leche por solo citar tres ejemplos (FDA/CFSAN, 2003; Barreto et al., 2009, 2010; Rodríguez et al., 2009, 2013). Tanto las características propias de cada alimento, como los procesos que se llevan a cabo durante su elaboración, son las dos principales fuentes de riesgos para su contaminación microbiana; sin embargo, el tipo de microorganismo causante de ETAs es otro elemento que no se puede soslayar (Rodríguez et al., 2015).

La principal causa de la salmonelosis no tifoidea, son los alimentos elaborados con huevo (CDC, 2021; OMS, 2018), sin embargo, el consumo de leche cruda también es causante de este tipo de brotes (Jayarao \& Henning, 2001). No obstante, se ha publicado que entre el 4,5 $\%$ y el $23 \%$ de los casos este agente llega al consumidor a través de derivados porcinos (Lee \& Middleton, 2003; Hald et al., 2004; Rodríguez et al., 2011a, b, 2013), los que se incrementan mundialmente cada año, y con ello las zoonosis asociadas. En los países que conforman la Unión Europea, estos productos, luego de los huevos y las aves, constituyen una importante fuente para la difusión de Salmonella (Rodríguez et al., 2011a, 2013).

Tabla 3. Brotes según el tipo de alimento

\begin{tabular}{|c|c|c|}
\hline Tipo de alimento & No. de brotes & $\%$ \\
\hline Cárnicos & 96 & 55.5 \\
\hline Repostería & 34 & 19.6 \\
\hline Lácteos & 15 & 8.7 \\
\hline Otros & 13 & 7.5 \\
\hline Pescados y derivados & 8 & 4.6 \\
\hline Elaborados de huevos & 7 & 4.1 \\
\hline Total & 173 & 100 \\
\hline
\end{tabular}

Fuente: Rodríguez et al., 2013.

\section{Prevención}

Entre las medidas que recomienda el programa conjunto FAO/OMS para asegurar la inocuidad alimentaria, son las Buenas Prácticas Agrícolas (BPA) o sea las técnicas aplicadas a los distintos cultivos y a la producción pecuaria para obtener productos satisfactorios en 
términos cualitativos y cuantitativos e inocuamente garantizados para el consumo (Díaz, 2008). Además, las BPA permiten tener un historial completo para el seguimiento del producto lo cual significa que se oferta al mercado algo de origen conocido y en forma transparente para satisfacer las exigencias de los consumidores (Reascos \& Castillo, 2019). La FAO promueve el uso de estas prácticas agrícolas en los proyectos y programas agrícolas que implementa.

Por otra parte, existen guías tecnológicas prácticas que la FAO promueve a través del Codex Alimentarius y pone a disposición de los usuarios tales como las Buenas Prácticas de Manufactura (BPM) que permiten controlar la higiene y sanidad durante todas las operaciones de los distintos procesos aplicados a los alimentos tales como refrigeración, congelación, envasado y cocción, deshidratado, azucarado, entre otros (FAO, 2009). Estas guías de BPM proporcionan los conocimientos técnicos básicos que se deben adoptar y aplicar a las materias primas en cada una de las operaciones a las que se someten durante la transformación industrial o preparación a nivel familiar de los alimentos para lograr una calidad e inocuidad garantizadas para el consumo (Estigarribia et al., 2019).

Las guías, tanto para las BPA como para las BPM, proveen normas y recomendaciones técnicas a seguir para obtener alimentos sanos y seguros. Estas normas y recomendaciones de las BPA y BPM deberían ser un material obligatorio de lectura y de consulta para quienes se dedican tanto a la producción como a la transformación o procesamiento de materias primas alimenticias. La OMS aconseja observar y adoptar las "Cinco Reglas de Oro de la OMS para la Inocuidad de Alimentos" tales como evitar la contaminación cruzada, utilizar materia prima y agua desde fuentes seguras, mantener correctamente la temperatura de los alimentos (sea de cocción, calentamiento y refrigeración) y la higiene personal del manipulador (FAO/OPS, 2016; OMS, 2015) .

\section{BIBLIOGRAFÍA}

Barreto G. (2007). Escherichia coli, últimos 122 años. Rev. Prod. Anim. (NÚMERO ESPECIAL): 55-67.

Barreto G., Sedrés, M., Rodríguez, H., Guevara, G. (2009). Comportamiento de los Brotes debidos a Enfermedades Trasmitidas por Alimentos (ETA) en la Provincia de Camagüey en el período 2000-2008.

Barreto-Argilagos, G.; Sedrés-Cabrera, M.; Rodríguez-Torrens, H.; Guevara-Viera, G. (2010). Agentes bacterianos asociados a brotes de Enfermedades Trasmitidas por Alimentos (ETA) 
en Camagüey, Cuba, durante el período 2000-2008. Revista Electrónica de Veterinaria, 11(2): 1-16.Disponible en: https://www.redalyc.org/pdf/636/63613118002.pdf

Bean N., Griffinm P., Goulding J. \& Ivey C. (1990). Foodborne disease outbreaks, 5-year summary, (1983-1987). J. Food Protect, 53: 711-728. https://doi.org/10.4315/0362-028X53.8 .711

Centros para el Control y Prevención de Enfermedades (CDC). (2021). La Salmonella y los huevos. Disponible en: https://www.cdc.gov/foodsafety/es/communication/salmonellahuevos.html

De la Hoz-Gómez, E.A. (2016). Comportamiento epidemiológico de las enfermedades transmitidas por alimentos (ETA) en el departamento de sucre durante el periodo 2012 2015. (Tesis de grado). Universidad Cooperativa de Colombia. 74 pp. Disponible en: https://repository.ucc.edu.co/bitstream/20.500.12494/742/1/Especializaci\%C3\%B3n\%20en $\% 20$ epidemiolog\%C3\%ADa.-.pdf

Díaz, A. (2008). Buenas Prácticas Agrícolas. Guía para Pequeños y Medianos Agroempresarios, Instituto Interamericano de Cooperación para la agricultura, IICA, Tegucigalpa, Honduras. 28 p. Disponible en: http://www.afipa.cl/web1/files/presentaciones/Buenas_Prcticas_Agrcolas_Importancia_par a_asegurar_la_inocuidad_de_los_alimentos.pdf

Dominguez, L.A. \& Oliver, C.R. (2007). Manipulador de alimentos: La importancia de la higiene en la elaboración y servicio de comidas. 2da Edición. Madrid. España, ES. p 38-40. ISBN: 978-84-9839-061-2

Estigarribia, G., Aguilar, G., Ríos, P., Ortíz, A., Martínez, P., \& Rios-González, C. M. (2019). Conocimientos, actitudes y prácticas sobre buenas prácticas de manufactura de manipuladores de alimentos en Caaguazú, Paraguay. Rev. salud pública Parag, 9(2): 2228. Disponible en: https://docs.bvsalud.org/biblioref/2019/12/1047048/2307-3349-rspp-902-22.pdf

Food and Drug Administration, FDA/CFSAN. (2003). Foodborne Pathogenic Microorganisms and Natural Toxins Handbook: The "Bad Bug Book." Center for Food Safety and Applied Nutrition, Food and Drug Admin., College Park, Md. Disponible en: https://www.fda.gov/media/83271/download

Garcia-Fernandez, A.J. (2020). Introducción a la Toxicología Alimentaria. Universidad de Murcia, España. 8 p. Disponible en: http://hdl.handle.net/10201/88641 
Gonzales-Flores, T. \& Rojas-Herrera, R.A. (2005). Enfermedades transmitidas por alimentos y PCR: prevención y diagnóstico. Salud pública de México, 47(5): 388-390. Disponible en: http://www.scielo.org.mx/pdf/spm/v47n5/28385.pdf

Gutiérrez, G. (2009). "Estudio de caso: Enfermedades Transmitidas por Alimentos en Nicaragua", en Enfermedades transmitidas por alimentos y su impacto socioeconómico, Organización de las Naciones Unidas para la Agricultura y la Alimentación. Roma, Italia. 159-190 p. Disponible en: http://www.fao.org/3/i0480s/i0480s06.pdf

Hald T., Vose, D., Wegener, H. C. \& Koupeev, T. (2004). A Bayesian approach to quantify the contribution of animal-food sources to human salmonellosis. Risk Anal; 24, 255-269. https://doi.org/10.1111/j.0272-4332.2004.00427.x

Hall, G.V., Kirk, M.D., Ashbolt, R., Stafford, R., Lalor, K. (2006). Frequency of infectious gastrointestinal illness in Australia, 2002: regional, seasonal and demographic variation. Epidemiol Infect, 134 (1): 111-8. https://doi.org/10.1017/S0950268805004656

Havelaar, A.H., Kirk, M.D., Torgerson, P.R., Gibb, H.J., Hald, T. et al. (2015). "World Health Organization global estimates and regional comparisons of the burden of foodborne disease in 2010”. Plos Med, 12 (12): 1-23. https://doi.org/10.1371/journal.pmed.1001923

Institute of Food Technologists (IFT). (2004). Bacteria Associated with Foodborne Diseases. Scientific Status Summary. Disponible https://seafood.oregonstate.edu/sites/agscid7/files/snic/bacteria-associated-with-foodbornediseases-scientific-status-summary-update2004-ift.pdf

Instituto Nacional de Salud, INS. (2018). Las enfermedades transmitidas por Alimentos-ETA. Boletín Epidemiológico Semanal: Semana epidemiológica 5223 al 29 de Diciembre de 2018. 31 pp. Disponible en: https://www.ins.gov.co/buscadoreventos/BoletinEpidemiologico/2018\%20Bolet\%C3\%ADn\%20epidemiol\%C3\%B3gico\%2 0semana\%2052.pdf

Jayarao B.M. \& Henning, D.R. (2001). Prevalence of foodborne pathogens in bulk tank milk. J. Dairy Sci. 84: 2157-2162. https://doi.org/10.3168/jds.S0022-0302(01)74661-9

Jones, K.E., Patel, N.G., Levy, M.A., Storeygard, A., Balk, D., Gittleman, J.L. \& Daszak, P. (2008). Tendencias mundiales en enfermedades infecciosas emergentes. Nature, 451 (7181): 990-993. doi: https://doi.org/10.1038/nature06536

Lee MB. \& Middleton, D. (2003). Enteric illness in Ontario, Canada, from 1997 to 2001. J Food Prot, 66: 953-961. https://doi.org/10.4315/0362-028X-66.6.953 
Más-Coma, M., Esteban J. \& Bargues, M. (1999). Epidemiology of human fascioliasis: a review and proposed new classification. Bulletin of the World Health Organization, 77:340-346. Disponible

en: https://www.ncbi.nlm.nih.gov/pmc/articles/PMC2557647/pdf/10327713.pdf

Mead P., Slutsker L., Dietz V., McCaig L., Bresee J., Shapiro C., Griffin P. \& Tauxe R. (1999). Food-related illness and death in the United States. Emerg Infect Dis., 5:607-625. https://dx.doi.org/10.3201/eid0505.990502

Olea, A., Díaz, J., Fuentes, R., Vaquero, A. y García M., "Vigilancia de brotes de enfermedades transmitidas por alimentos en Chile”, Rev Chilena Infectol. 2012, 29 (5): 504-510. http://dx.doi.org/10.4067/S0716-10182012000600004

Organización de las Naciones Unidas para la Agricultura y la Alimentación, FAO. (2009). Enfermedades transmitidas por alimentos y su impacto socioeconómico: Estudios de caso en Costa Rica, El Salvador, Guatemala, Honduras y Nicaragua. Informe técnico sobre ingeniería agrícola y alimentaria. 194 pp. Disponible en: http://www.fao.org/3/i0480s/i0480s.pdf

Organización de las Naciones Unidas para la Agricultura y la Alimentación/Organización Panamericana de la Salud. FAO/OPS. (2016) Educación en inocuidad de alimentos. Washington D. C., Estados Unidos.

Organización Mundial de la Salud, OMS. (2018). Salmonella (no tifoidea). Disponible en: https://www.who.int/es/news-room/fact-sheets/detail/salmonella-(nontyphoidal)\#: :text=Por\%20lo\%20general\%2C\%20las\%20personas,las\%20hortalizas\%20c ontaminadas\%20por\%20esti\%C3\%A9rcol.

Organización Mundial de la Salud, OMS. (2002). Food safety and foodborne illness Fact sheet $\mathrm{N}^{\circ} 237: 1-4$.

Organización Mundial de la Salud, OMS. (2006). WHO Consultation to Develop a Strategy to Estimate the Global Burden of Foodborne Diseases. 56 pp. Disponible en: https://www.who.int/foodsafety/publications/foodborne_disease/fbd_2006.pdf

Organización Mundial de la Salud, OMS. (2007). Manual sobre las cinco claves para la inocuidad de los alimentos. 32 p. Disponible en: https://www.who.int/foodsafety/publications/consumer/manual_keys_es.pdf

Organización Mundial de la Salud, OMS. (2015). Enfermedades de transmisión alimentaria. Washington D. C., Estados Unidos; 2015. 
Organización Mundial de la Salud, OMS. (2015). WHO estimates of the global burden of foodborne diseases. Foodborne disease burden epidemiology reference group 2007-2015. 265 pp.

Disponible

en: https://apps.who.int/iris/bitstream/handle/10665/199350/9789241565165_eng.pdf?sequenc $\mathrm{e}=1$

Organización Panamericana de la Salud, OPS. (2019). Análisis de peligros y puntos críticos de control (HACCP). 171 pp. Disponible en: https://www.paho.org/es/documentos/analisis-peligros-puntos-criticos-control-haccp

Pinillos, M.A.; Gómez, J.; Elizalde, J. \& Dueñas, A. (2003). Intoxicación por alimentos, plantas y setas. ANALES Sis San Navarra, 26(1): 243-263. Disponible en: http://scielo.isciii.es/pdf/asisna/v26s1/quince.pdf

Reascos-Pardo, J.L., \& Castillo-Criollo, F. (2019). Buenas prácticas agrícolas. Observatorio de la Economía Latinoamericana. 5 pp. Disponible en: https://www.eumed.net/rev/oel/2019/03/buenas-practicas-agricolas.html

Riverón RL. (1999). Fisiopatología de la diarrea aguda. Rev Cubana Pediatr; 71 (2): 86-115. Disponible en: http://scielo.sld.cu/pdf/ped/v71n2/ped05299.pdf

Roberts, J.A. \& Sockett, P.N. (1994). The socio-economic impact of human Salmonella enteritidis infection. Int J Food Microbiol, 21 (1-2): 117-29. https://doi.org/10.1016/01681605(94)90205-4

Rodríguez H., Barreto, G., Sedrés, M., Bertot, J., Martínez, S., Guevara, G. (2011). Los alimentos de origen porcino: vehículos predominantes en las salmonelosis camagüeyanas. Rev. Prod. Anim; 23 (2): 109-112.

Rodríguez H., Barreto, G., Sedrés, M., Guevara, G. (2009). Comportamiento de las enfermedades transmitidas por alimentos (ETA) en la provincia de Camagüey según el tipo de muestra analizado. Rev. Prod. Anim; 20 (1): 81-88. 87. Disponible en: https://revistas.reduc.edu.cu/index.php/rpa/article/view/2977

Rodríguez H., Barreto, G., Sedrés, M., Guevara, G., Bertot, J. (2013). Los cárnicos: vehículos principales en los brotes de enfermedades alimentarias bacterianas en Camagüey, Cuba (Meat products: main vehicles of the bacterial foodborne outbreaks in Camagüey, Cuba). Revista electrónica de Veterinaria, 14 (3). Disponible en: http://www.veterinaria.org/revistas/redvet/n030313/031307.pdf.

Rodríguez H., Sedrés, M., Barreto, G., Guevara, G., Bertot, J., Martínez, S. (2011). Serogrupos de Salmonella en alimentos y coprocultivos. Rev. Prod. Anim; 23 (2): 121-124. 
Rodríguez-Torrens, H.; Barreto-Argilagos, G.; Sedrés-Cabrera, M.; Bertot-Valdés, J.; Martínez-Sáez, S.; Guevara-Viera, G. (2015). Las enfermedades transmitidas por alimentos, un problema sanitario que hereda e incrementa el nuevo milenio Revista Electrónica de Veterinaria, 16(8):1-27. https://www.redalyc.org/pdf/636/63641401002.pdf

Rosas, MR. (2007). Contaminaciones alimentarias. Cuadros principales, tratamiento y prevención. Nutrición, 25(6): 95-100. Disponible en: https://www.elsevier.es/es-revistaoffarm-4-pdf-13107676

Schmidt, R.H., Goodrich, RM., Archer, D.L., Schneider, KR. (2003). General Overview of the Causative Agents of Foodborne Illness. This document is FSHN033, one of a series of the Food Science and Human Nutrition Department, Florida Cooperative Extension Service, IFAS, University of Florida. Publication: February 2003. Disponible en: http://edis.ifas.ufl.edu

Schneider K.R., Parish M.E., Goodrich R.M. \& Cookingham, T. (2004). Preventing Foodborne Illness: Bacillus cereus and Bacillus anthracis. Florida Cooperative Extension Services, EDIS FSHN036.

Vasquez de Plata, G. (2003). La Contaminación de los Alimentos, un Problema por Resolver. Rev. Salud UIS, 35(1): 48-57. Disponible en: https://revistas.uis.edu.co/index.php/revistasaluduis/article/view/728/1014

Zúñiga-Carrasco, I.R. \& Caro-Lozano, J. (2017). Enfermedades transmitidas por los alimentos: una mirada puntual para el personal de salud. Enfermedades transmitidas por los alimentos, 37(3): 95-104. https://www.medigraphic.com/pdfs/micro/ei-2017/ei173e.pdf 